Avascular necrosis (AVN) refers to the death of osteocytes and osteoblasts. $\mathrm{AVN}$ is encountered mostly among men in their $4^{\text {th }}$ or $5^{\text {th }}$ decade of life. The basic treatment for osteonecrosis is surgery, and the choice of procedure is based on preoperative staging. AVN is a very rare complication in radiotherapy (RT), but it presents an important clinical problem, still underestimated in oncological practice.

Case report: This report documents an extensive symmetric avascular necrosis with acetabular protrusion and femoral head deformities in a 71-yearold woman with unresectable, poorly differentiated adenocarcinoma of the recto-sigmoid region, after therapeutic pelvic irradiation. The pelvic radiography, performed 24 months after the RT, due to bilateral hip pain, revealed advanced acetabular protrusions, with secondary deformities of both femoral heads.

It is necessary to take into account the bone structures, among organs at risk (OAR), involved in irradiation fields. The detailed analysis of the dose distribution, and the use of collimators, allow a decrease in the total RT dose to OAR. Osteoradionecrosis risk awareness, early diagnosis, adequate management and prompt, appropriate treatment could probably protect patients against long-term morbidity.

Key words: osteoradionecrosis, aseptic necrosis, radiotherapy.

Contemp Oncol (Pozn) 2014; 18 special issue DOI: $10.5114 /$ wo.2014.40604

\section{Avascular osteonecrosis of the hip in a patient after adjuvant radiotherapy for rectal cancer}

\author{
Łukasz Michalecki, Dorota Gabryś, Grzegorz Głowacki, Hubert Urbańczyk, \\ Jerzy Wydmański
}

Department of Radiotherapy, Maria Skłodowska-Curie Memorial Cancer Centre and Institute of Oncology, Gliwice Branch, Gliwice, Poland

\section{Introduction}

The term aseptic or avascular necrosis (AVN) refers to the death of osteocytes, caused by insufficient oxygen delivery, due to abnormal local circulation. Its development is related to the influence of different adverse factors on bone structure such as corticosteroids, alcohol abuse, chemotherapy or, as recently postulated, thrombophilia. Bones such as the femoral or arm head as well as small bones of the hands and feet receive their blood supply from single, terminal blood vessels only, and thus are more vulnerable to AVN, compared with the areas supplied by wide blood vessel networks derived from various overlapping sources [1-5].

The male-to-female ratio for AVN is about $4: 1$ mostly among people in their $4^{\text {th }}$ or $5^{\text {th }}$ decade of life. However, according to the literature, there is a growing incidence of osteonecrosis among children $[2,6]$. It has been assessed that at least $50 \%$ of atraumatic hip osteonecrosis have bilateral involvement. Other bones such as shoulder, knee and talus may also be involved in atraumatic forms of osteonecrosis [3].

The most common cause of mechanical blockade to blood flow, leading to osteonecrosis, is trauma including fraction with bone displacement or osteoporotic fraction. Osteonecrosis, especially of the femoral head, can also occur in pregnancy [1, 7]. An increase in the pressure within the bone marrow cavity, as a result of haemorrhage or cellular hyperproliferation (Gaucher's disease or in hypertrophy of adipocytes), can also lead to compression of blood vessels and dysfunction in local blood flow $[8,9]$.

Avascular necrosis can be enhanced by disorders associated with damage of blood vessel walls due to inflammatory processes or autoimmune diseases of connective tissue such as systemic lupus erythematosus (SLE), scleroderma or vasculitis [1].

Non-traumatic factors are related to about $90 \%$ of AVN cases and they include alcohol overuse, long-term corticosteroid therapy and chemotherapy $[1,10]$.

After radiotherapy $A V N$ is a rare and poorly understood phenomenon. However, considering the very common use of radiotherapy (30-40 thousand patients per year in Poland), this issue definitely requires more attention since this late toxicity problem is still underestimated by many physicians.

The pathogenesis of AVN is multifactorial and, depending on the irradiated area, it can involve almost the entire skeleton. The Rubin \& Cassaret theory explains the effect of irradiation on microvasculature as a result of slow vascular leakage of the protein-rich fluid leading to fibrosis caused by the accumulation of fibroblasts and macrophages [11-13].

Tolerance doses (TD5/5 - TD50/5) represent the dose of radiation that could cause no more than $5 \%$ and $50 \%$, respectively, of severe complica- 
tion rate within 5 years after irradiation. Referring to bone structures, they range from 20 to 30 Gy for single and 65 to 70 Gy for fractionated doses. As the volume of irradiated bone or cartilage decreases, the dose to produce $5 \%$ or $50 \%$ of complications increases. Simultaneously, the patient's bone structure has crucial meaning, as well as its localization and the patient's age. Tolerable doses for different types of tissues have been established empirically, based on the frequency of reported early and late toxicity, in patients receiving standard RT, using a fraction of 2 Gy, once daily. For this reason using new, accelerated or hypofractionated RT schedules (above these limits) can be related to increased toxicity risk of the normal tissues $[11,14,15]$.

A differential diagnosis of bone pain in oncology patients receiving RT should always include AVN. A combination of risk factors for bone destruction amplifies the incidence of AVN in this group of patients.

Aseptic necrosis can develop insidiously over several months, before its symptomatic manifestation appears clinically. It depends on the location and advancement (stage) of AVN. The most common symptom is deep and throbbing pain with varying intensity and patient mobility impairment. It is also worse at night. Locking or a popping sensation with motion is often reported. This process can simultaneously involve one or more locations. A history of radiation therapy, trauma, steroid use, alcohol abuse and other risk factors should be sought in order to distinguish symptoms from metastases or oncologic disease progression $[3,10]$.

Medical management of a patient with histopathologically confirmed diagnosis of AVN depends on the disease site and stage of clinical advancement, as well as the age and general condition of the patient.
In cases of femoral head necrosis, stages I and II are reversible, while stages III and IV require surgical treatment $[1,16]$. Additionally pentoxifylline, tocopherol (vitamin E), statins, anticoagulants and prostacyclin analogues (iloprost) can be considered as therapeutic options for AVN $[13,16]$.

Recently, tissue hypoxia was accepted as the primary cause of AVN, and this led to the use of hyperbaric oxygen $(\mathrm{HBO})$ for both treatment and prevention of complications of radiotherapy. However, the benefit of HBO has not been validated [12]. According to a consensus of the European Committee of Hyperbaric Medicine (ECHM) and the Underwater and Hyperbaric Medicine Society (UHMS), it is indicated that the osteoradionecrosis of the mandible (group I) requires an immediate transfer to a specialist hyperbaric oxygen ( $\mathrm{HBO}$ ) therapy centre; in cases of AVN in other locations (group II) the HBO therapy is less urgent [4].

\section{Case report}

A 71-year-old woman diagnosed with unresectable, poorly differentiated adenocarcinoma of the recto-sigmoid region developed bilateral avascular necrosis of the hip after receiving hyper fractionated radiotherapy, using 3D technique, with photon beam $\times 20 \mathrm{mV}$ and a fractionated dose of 1.5 Gy, 2 times daily, with 6-hour interval. The first part of irradiation - to the dose of 42 Gy was delivered to rectal tumour and regional lymph nodes. Subsequently a total dose of 66 Gy was delivered to the tumour area. The dose above 60 Gy covered $25 \%$ and $10 \%$ of the right and left femoral head, respectively. The patient completed treatment without any significant acute post-radiation toxicity. However, 24 months after the

Table 1. International (ARCO) Classification of Osteonecrosis of the Femoral Head [17]

\begin{tabular}{|c|c|}
\hline Stage & Characteristics \\
\hline 0 & Bone biopsy results consistent with avascular necrosis; normal findings on all other tests \\
\hline । & $\begin{array}{l}\text { Positive scintiscan, magnetic resonance image or both; lesions subdivided into medial, central or lateral depending } \\
\text { on location of involvement of femoral head }\end{array}$ \\
\hline I-A & $<15$ per cent involvement of femoral head* \\
\hline I-B & 15-30 per cent involvement of femoral head* \\
\hline I-C & $>30$ per cent involvement of femoral head* \\
\hline II & $\begin{array}{l}\text { Radiographic abnormalities (mottled appearance of femoral head, osteosclerosis, cyst formation and osteopenia); no signs } \\
\text { of collapse of femoral head on radiographs or computerized tomography scan; positive scintiscan and magnetic resonance } \\
\text { image; no changes in acetabulum; lesions subdivided into medial, central or lateral depending on location of involvement } \\
\text { of femoral head }\end{array}$ \\
\hline II-A & $<15$ per cent involvement of femoral head* \\
\hline II-B & 15-30 per cent involvement of femoral head* \\
\hline II-C & > 30 per cent involvement of femoral head* \\
\hline $\begin{array}{l}\text { III } \\
\text { III-A } \\
\text { III-B } \\
\text { III-C }\end{array}$ & $\begin{array}{l}\text { Crescent sign; lesions subdivided into medial, central or lateral depending on location of involvement of femoral head } \\
<15 \text { per cent involvement of femoral head } \\
15-30 \text { per cent involvement of femoral head } \\
>30 \text { per cent involvement of femoral head }\end{array}$ \\
\hline IV & $\begin{array}{l}\text { Articular surface flattened radiographically, and joint space shows narrowing; changes in acetabulum with evidence } \\
\text { of osteosclerosis, cyst formation and marginal osteophytes }\end{array}$ \\
\hline
\end{tabular}

${ }^{*}$ As determined on magnetic resonance imaging

${ }^{\dagger}$ As determined on anteroposterior and lateral radiographs. The percentage given for the crescent sign refers to the percentage of the femoral head that the crescent sign subtends on the anteroposterior and lateral radiographs. 

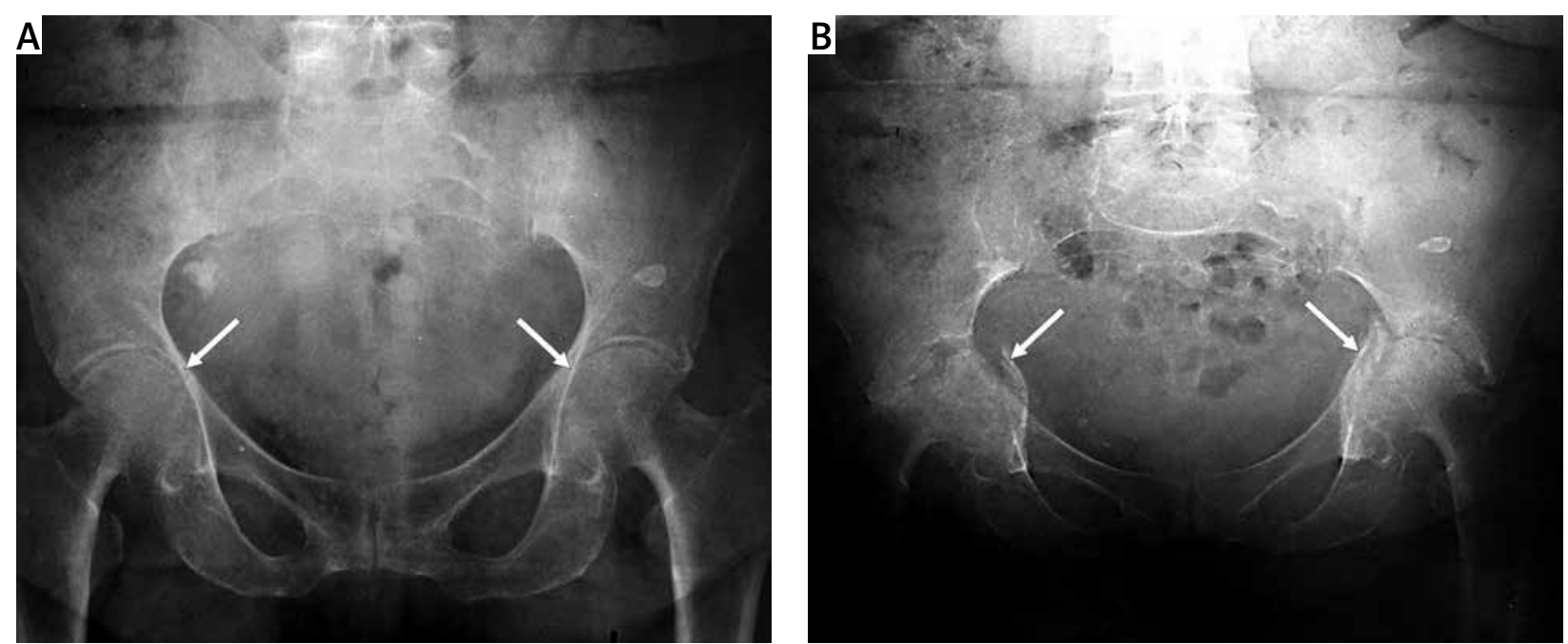

Fig. 1. Pelvis and hips radiographs of a 71-year-old woman with unresectable rectal adenocarcinoma - before (A) and after radiotherapy (RT) (B) A) performed before RT

B) performed 24 months after RT; protrusions with secondary deformities of both femoral heads - stage IV, according to ARCO classification

termination of RT, the patient started complaining about pain in her hips. On radiology imaging, including bilateral hip radiograph and pelvic computerized tomography (CT), advanced bilateral acetabular protrusions with secondary deformities of both femoral heads were found stage IV according to ARCO (Association Research Circulation Osseous) classification, Table 1 [17] (Fig. 1A). There were no changes in the patient's radiographs performed before radiotherapy (Fig. 1B).

The patient was referred for an orthopaedic evaluation to establish a further clinical pathway and eventual treatment possibilities including bilateral hip arthroplasty.

\section{Summary}

Good results of pharmacologic treatment in early disease stages and a broad range of orthopaedic and rehabilitation procedures, improving functional capacity of limbs, contribute to better quality of life and allow faster adjustment of the patient to new conditions of life. Prediction of the consequences of applied RT, including an analysis of multiple risk factors for AVN as well as prompt implementation of preventive or therapeutic methods, are necessary for safe and effective management. It is mandatory that the bone structures located within the RT target area should be considered as critical organs. Detailed analysis of RT beam configuration and dose distribution as well as using collimator shields can effectively reduce the toxicity delivered to organs. In summary, osteoradionecrosis risk awareness, regular follow-ups with diagnostic imaging and, in the case of positive diagnosis of first symptoms, immediate, comprehensive treatment could help to protect patients from irreversible disability.

All authors certify they have no financial or personal relationships with other people or organisations that could inappropriately influence (bias) their work.

\section{References}

1. Sawicka-Żukowska M, Krawczuk-Rybak M, Muszyńska-Rosłan K. Martwica aseptyczna - niedoceniany problem w onkologii dziecięcej. Pol Merk Lek 2006; 21: 291-4.

2. Aaron RK. Osteonecrosis: etiology, pathophysiology, and diagnosis. In: Callahan JJ, Rosenberg AG, Rubash HE (eds.). The Adult Hip. Lippincott-Raven Publishers, Philadelphia 1998; 451-66.

3. Levine M, Rajadhyaksha A, Mont M. Hip osteonecrosis. eMedicine Journal, Orthopedic Surgery, 2009. Available at: http://emedicine. medscape.com/article/1247804-overview.

4. Lyons A, Ghazali N. Osteoradionecrosis of the jaws: current understanding of its pathophysiology and treatment. Br J Oral Maxillofac Surg 2008; 46: 653-60.

5. Cenni E, Fotia C, Rustemi E, Yuasa K, Caltavuturo G, Giunti A, Baldini N. Idiopathic and secondary osteonecrosis of the femoral head show different thrombophilic changes and normal or higher levels of platelet growth factors. Acta Orthop 2011; 82: 42-9.

6. Pavelka K. Osteonecrosis. Baillieres Best Pract Res Clin Rheumatol 2000; 14: 399-414.

7. Vaidya S, Saika S, Sirohi B, Pai S, Advani S. Avascular necrosis of bone - a complication of aggressive therapy for acute lymphoblastic leukemia. Acta Oncol 1998; 37: 175-7.

8. Nikolopoulos KE, Papadakis SA, Kateros KT, Themistocleous GS, Vlamis JA, Papagelopoulos PJ, Nikiforidis PA. Long-term outcome of patients with avascular necrosis, after internal fixation of femoral neck fractures, injury. Int I Care Injured 2003; 34: 525-8.

9. Pajączkowski JA. The stubborn hip: idiopathic avascular necrosis of the hip. J Manipulative Physiol Ther 2003; 26: 107.

10. Biswas TK, Biswas S. Avascular necrosis in rheumatology practice. APLAR J Rheumatol 2004; 7: 175-8.

11. Niewald M, Barbie O, Schnabel K, Engel M, Schedler M, Nieder C, Berberich W. Risk factors and dose-effect relationship for osteoradionecrosis after hyperfractionated and conventionally fractionated radiotherapy for oral cancer. Br J Radiol 1996; 69: 847-51.

12. Herman MP, Kopetz S, Bhosale PR, et al. Sacral insufficiency fractures after preoperative chemoradiation for rectal cancer: incidence, risk factors and clinical course. Int I Radiation Oncology Biol Phys 2009; 74: 818-23.

13. Delanian S, Lefaix JL. Complete healing of severe osteoradionecrosis with treatment combining pentoxifylline, tocopherol and clodronate. Br J Radiol 2002; 75: 467-9.

14. Gasińska A. Biologiczne podstawy radioterapii. Kraków 2001; 70-1.

15. Perez CA, Halperin HC, Brady LW. Principles and Practice of Radiation Oncology. Lipincott Williams \& Wilkins, Philadelphia 2007; 157-62. 
16. Petty W. Osteonecrosis: Strategies for treatment. In: The Adult Hip. Callahan, Rosenberg AG, Rubashed HE (eds.). Lippincott-Raven Publishers, Philadelphia 1998; 467-84.

17. ARCO (Association Research Circulation Osseous). Committee on terminology and classification. ARCO NEWS, 1992, 4, 41-46. Reproduced in Mont and Hungerford. J Bone Joint Surg 1995; 77-A, No. 3.

\section{Address for correspondence}

Łukasz Michalecki

Department of Radiotherapy

Maria Skłodowska-Curie Memorial Cancer Centre

and Institute of Oncology

Gliwice Branch

Gliwice, Poland

e-mail: lukaszmichalecki@gmail.com

Submitted: 4.01 .2012

Accepted: 3.10 .2012 